Original Research Paper

\title{
A Novel Modulation Technique for 5G Mobile Communication System
}

\author{
${ }^{1}$ Arun Kumar and ${ }^{2}$ Manisha Gupta \\ ${ }^{I}$ Department of ECE, Jecrc University, Jaipur, India \\ ${ }^{2}$ Department of Physics, Jecrc University, Jaipur, India
}

\author{
Article history \\ Received: 10-09-2015 \\ Revised: 18-09-2015 \\ Accepted: 21-09-2015 \\ Corresponding Author: \\ Arun Kumar \\ Department of ECE, Jecrc \\ University, Jaipur, India \\ Email: arun.kumar1986@live.com
}

\begin{abstract}
By the year 2020, the data traffic can be approximately increase by $30 \%$, which cannot support by existing mobile technologies. Hence, there is a requirement of next generation wireless communication (5G) which aim to provide a high capacity, data-rate at the speed of 1 Tbps. By selecting a better modulation technology, the required capacity and datarate can be achieved. In this study we design aprototype filter for multicarrier modulation candidates of 64-subchannels for $5 \mathrm{G}$ mobile communication system: Orthogonal Frequency Division Multiplexing (OFDM) often used in 4G mobile and Filter Band Multi-Carrier (FBMC) a promising candidate for $5 \mathrm{G}$ mobile communication system. We judge the magnitude response of prototype filter for OFDM and FBMC. Magnitude response for overlapping factor $(\mathrm{K}=2,3,4)$ is also evaluated. Finally the constellation plot clearly defines the transmission scheme of FBMC and OFDM. FBMC proof to be preeminent method and outperforms OFDM due to the fact that FBMC do not use (CP) cyclic prefix and wide guard band un-like OFDM.
\end{abstract}

Keywords: OFDM, FBMC, CP, Magnitude Response

\section{Introduction}

The successor of $3 \mathrm{G}$ technology is $4 \mathrm{G}$ where Orthogonal Frequency Division Multiplexing (OFDM) is used as a modulation technology and it gives an Ultra Broad Band access for a mobile device with more capacity and data-rate and better service as compared to $3 \mathrm{G}$ Mobile. In OFDM System, the bandwidth is divided into a number of subcarriers which are orthogonal to each-others which in-turns increase the capacity, speed of a system and Inter-symbol-Interference is also reducing OFDM by inserting a Cyclic prefix between the symbols which is regarded as one of the biggest problems in $3 \mathrm{G}$ technology. The OFDM also provides a flat channel response of a system. Peak-Average-Power-Ratio (PAPR) is considered to be the biggest hurdle of OFDM which reduces the efficiency of a System. Due to the disadvantages like PAPR and cyclic prefix (which reduces the bandwidth efficiency), OFDM is not considered for Fifth Generation Mobile Communication system (Kumar and Gupta, 2015). Filter Band-Multi-Carrier System (FBMC) is considered as one of the most suitable modulation schemes for $5 \mathrm{G}$ Mobile. The implementation of FMBC is similar to the OFDM but it do-not use cyclic prefix, hence bandwidth efficiency is achieved (Ndo et al., 2012).

\section{Filter Band Multi-Carrier (FBMC)}

It is a multi-carrier modulation techniques in which prototype filter is used to eliminate the effect of ISI, ICI. The FBMC is implemented on the basis of OFDM except excluding the use of cyclic-prefix between the symbols due to which more bandwidth utilization is achieved which was wasted during the case of OFDM. The white TV space spectrum which is currently not utilized by anyone will be utilized by the FBMC receiver because FBMC can control the out of band interference level (Fang et al., 2013). In this technique, a bank of filter is used through which a set of parallel data is transmitted. The adjacent leakage and localization of frequency can be controlled by using an appropriate prototype filter. Due to the flexibility in the frequency domain of FBMC it is better suited for TVWS and spectrum usage. The channel delay spread can easily handle by FBMC also fragmented spectrum accessing is achieved-able (Berg et al., 2014a). The de-modulation signal of FBMC contained an interference which is caused adjacent data-transmission in the time frequency domain. This has become an important issue while implementing FBMC with ML Equalizer which can be reduced by combining FBMC with OFDM. The FBMC 
scheme also over-come the internal interference like inter-carrier-interference that comes from neighboring sub cell and ISI that comes from symbols (Zakaria and Le Ruye, 2010). The original signal in FBMC can recover by separation of frequency component and by recombination of the signal component. The authors of this paper have used relax synchronization in FBMC to reduce the signal overhead. The proposed system was implemented by using an FPGA Kintex-7XC7K325T. Finally, the authors have concluded that FBMC outperforms OFDM for high order modulation scheme (Berg et al., 2014b). The authors of this work ha compare FBMC and Cyclic prefix based OFDM to evaluate the BER probability under an AWGN channel and conclude that the performance of both systems is similar when all conditions is satisfied, however FBMC gives less out band power leakage as compare to OFDM (He and Schmeink, 2015).

\section{Implementation of FBMC}

The transmitter side the baseband equivalent of a discrete time FBMC signal as follows:

$$
t(m)=\sum_{k=0}^{M-1} \sum_{n \in Z}^{\phi} a_{k, n} g\left[m-\frac{n M}{2}\right] e^{i \frac{2 \pi}{M}} k\left(m-\frac{D}{2}\right) e^{i \varphi_{k, n}}
$$

Where:

$\mathrm{M}=$ Even no. Of subcarriers

$\mathrm{D}=$ Delay term depends on the length of the prototype filter $\mathrm{g}(\mathrm{m})$

$\phi_{\mathrm{k}, \mathrm{n}}=$ An additional phase term

$\mathrm{a}_{\mathrm{k}, \mathrm{n}}=$ Real valued transmitted symbols

The real and imaginary parts are driven by the phase term $\phi_{\mathrm{k}, \mathrm{n}}$ given by:

$$
\varphi_{k, n}=\varphi_{0}+\frac{\pi}{2}(n+k)-\pi n k
$$

Here $\phi 0=0$, we can write the Equation 1:

$$
t(m)=\sum_{k=0}^{M-1} \sum_{n \in Z} a_{k, n} g_{k, n}[m]
$$

where, $g_{k, n}[m]=$ Shifted version of $g[m]$ in time and frequency.

In the case of channel, the demodulated symbol over the $\mathrm{k}^{\text {th }}$ sub-carrier and the $\mathrm{n}^{\text {th }}$ instant is determined using the inner product of $t(m)$ and $g_{k, n}[m]$ :

$$
\begin{aligned}
& r_{k^{\prime}, n^{\prime}}=\left\langle s, g_{k^{\prime} n^{\prime}}\right\rangle=\sum_{m=-\infty}^{+\infty} s[m] g_{k^{\prime} n^{\prime}}^{*}[m] \\
& =\sum_{m=-\infty}^{+\infty} \sum_{k=0}^{M-1} \sum_{n \in Z} a_{k, n} g_{k, n}[m] g_{k^{\prime} n^{\prime}}^{*^{\prime}}[m]
\end{aligned}
$$

From this equation the transmit-receive antenna impulse response can be derived assuming null data except one frequency position $\left(\mathrm{k}_{0}, \mathrm{n}_{0}\right)$ where a unit impulse is applied. The Equation 4 becomes:

$$
\begin{aligned}
& r_{k^{\prime} n^{\prime}}=\sum_{m=-\infty}^{+\infty} g_{k 0, n 0}[m] g_{k^{\prime} n^{\prime}}^{*^{\prime}}[m] \\
& r_{k^{\prime} n^{\prime}}=\sum_{m=-\infty}^{+\infty} g\left[m-\frac{n 0 M}{2}\right] g\left[m-\frac{n^{\prime} M}{2}\right] \cdot e^{i \frac{2 \pi}{M}}\left(k 0-k^{\prime}\right) \\
& \left(m-\frac{D}{2}\right) e^{i\left(\varphi k 0, n 0-\varphi k^{\prime} n^{\prime}\right)}
\end{aligned}
$$

By using the substitution of $\mathrm{m}$ by $\mathrm{m}+\mathrm{n} 0 \mathrm{M} / 2$ and denoting $\Delta \mathrm{n}=\mathrm{n}$ '- $\mathrm{n} 0, \Delta \mathrm{k}=\mathrm{k}$ '-k0, we obtain:

$$
\begin{aligned}
& r_{k^{\prime} n^{\prime}}=\sum_{m=-\infty}^{+\infty} g[m] g\left[m-\frac{\Delta \mathrm{nM}}{2}\right] \\
& e^{i \frac{2 \pi}{M}} \Delta \mathrm{k}\left(\frac{\mathrm{D}}{2}-\mathrm{m}\right) \cdot e^{i \pi(\Delta \mathrm{k}+\mathrm{k} 0) \Delta \mathrm{n}} e^{-i \pi(\Delta \mathrm{k}+\Delta \mathrm{n})}
\end{aligned}
$$

\section{Orthogonal-Frequency-Division- Multiplexing}

Due to the disadvantage in CDMA like Inter-SymbolInterference, high power circuit requirement and crosstalk, the CDMA is overtaken by OFDM. The OFDM offers a high data-rate and also minimize the ISI by inserting a cyclic prefix between the symbols. Basically, An OFDM is a multi-carrier technique where available bandwidth is divided into the number of subcarriers which are orthogonal to each-others due to which power consumption is reduced, bandwidth is efficiently utilized and also provide the flat response of a channel. In OFDM, the digital data are encoded in a subcarriers. One of the disadvantages of OFDM is Peak-Average-Power-ratio. For example, If PAPR of a system is $10 \mathrm{~dB}$, then in order to transmit a 1 watt of the signal, we need 10 watts of a signal. In this way, the efficiency of the system is reduced (Han and Lee, 2005). The first multi carrier technique was proposed by (Chang, 1996). Doelz et al. (1957) had designed a multi carrier system for a single side band channel. Septh et al. (1999) had designed an OFDM receiver and has demodulated the signal and deliver the soft information to an outer receiver for decoding. Lu and Wang (2000) work he has considered Space Time Codded OFDM and his result shows a significant improve in the performance of OFDM by efficiently exploiting the spatial diversity and selective fading.

\section{Basic OFDM System Model}

Let us consider a complex symbol to be transmitted by using an OFDM technique. The modulated signal can be represented by following mathematical expression:

$Z n(t)=\sum_{k=0}^{n-1} \mathrm{Z}(\mathrm{n}), \mathrm{Ke}^{\mathrm{j} 2 \pi \mathrm{k} \Delta \mathrm{ft}}$ 
Ts $=$ Symbol duration

$\Delta \mathrm{f}=$ Sub carrier spacing

$\mathrm{N}=$ Number of Sub-channel

With the orthogonality condition Ts. $\Delta \mathrm{f}=1$, the transmitted symbol $Y_{n}, k$ can be received by the receiver as described in the following equation:

$\mathrm{Yn}(\mathrm{k})=\frac{1}{\mathrm{Ts}} \int_{0}^{T S Y n(t) e-j 2 \pi k \Delta f t} d t$.

With the cyclic Prefix the transmitted signal can be written as:

$$
\mathrm{T}=\mathrm{T}_{\mathrm{g}}+\mathrm{T}_{\mathrm{s}}
$$

Therefore:

$$
\mathrm{Zn}(\mathrm{t}+\mathrm{Ts})=\sum_{N-1}^{k=0} \mathrm{Yn}, \mathrm{K} e^{J 2 \pi k \Delta f(t+T s)}
$$

The impulse response of a channel is given by the following equation:

$$
\mathrm{i}(\mathrm{t})=\mathrm{bi} \delta(\mathrm{t}-\mathrm{ti})
$$

where, bi and ti are delay and complex amplitude ${ }^{2}$ of $\mathrm{i}^{\text {th }}$ path.

The received signal is given by:

$V n(t)=\sum_{i}^{\phi} \mathrm{Zn} x^{k}(\mathrm{t}-\mathrm{ti})+\mathrm{n}$

where, $\mathrm{n}$ is the noise of a signal.

\section{Results}

The result and simulation is simulated on the basis of Mat-Lab Simulation.

The plot of magnitude response of OFDM and FBMC is shown in above Fig. 1. As shown above denoted by green line, the magnitude of OFDM is constant throughout the frequency which means it is more affected by Peak-Average-Power-Ratio which reduce the efficiency of OFDM system whereas initially, the response of FBMC is more than $40 \mathrm{~dB}$ and it reduces with the increase of frequency.

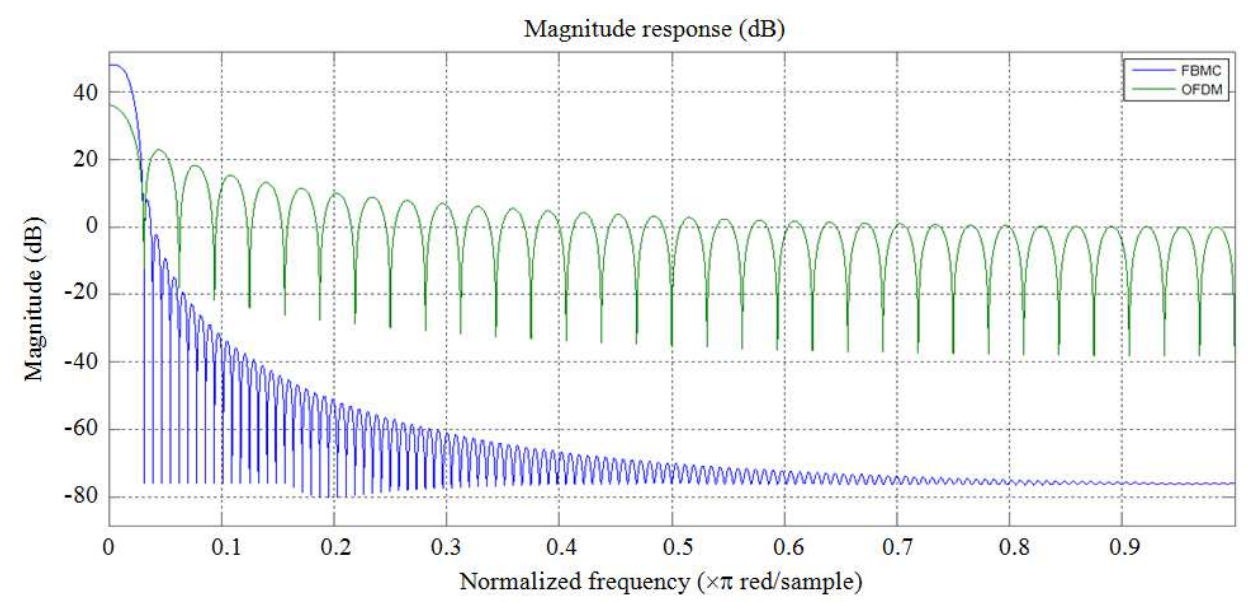

Fig. 1. Magnitude response of OFDM and FBMC

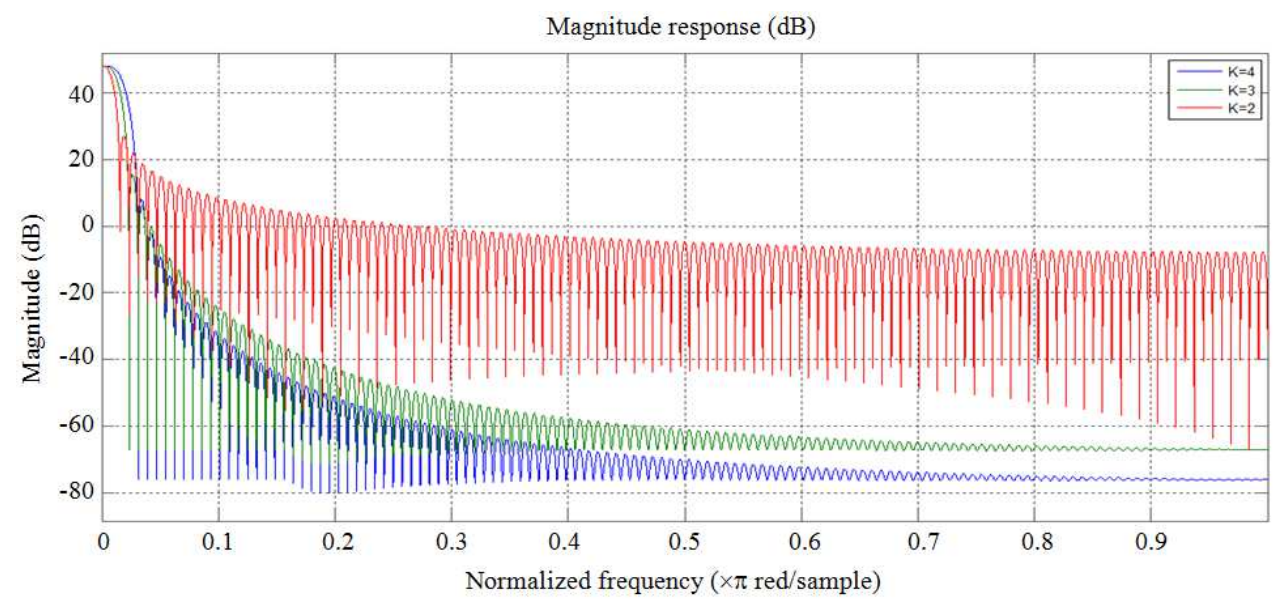

Fig. 2. Magnitude response for overlapping factor $(\mathrm{K}=2,3,4)$ 


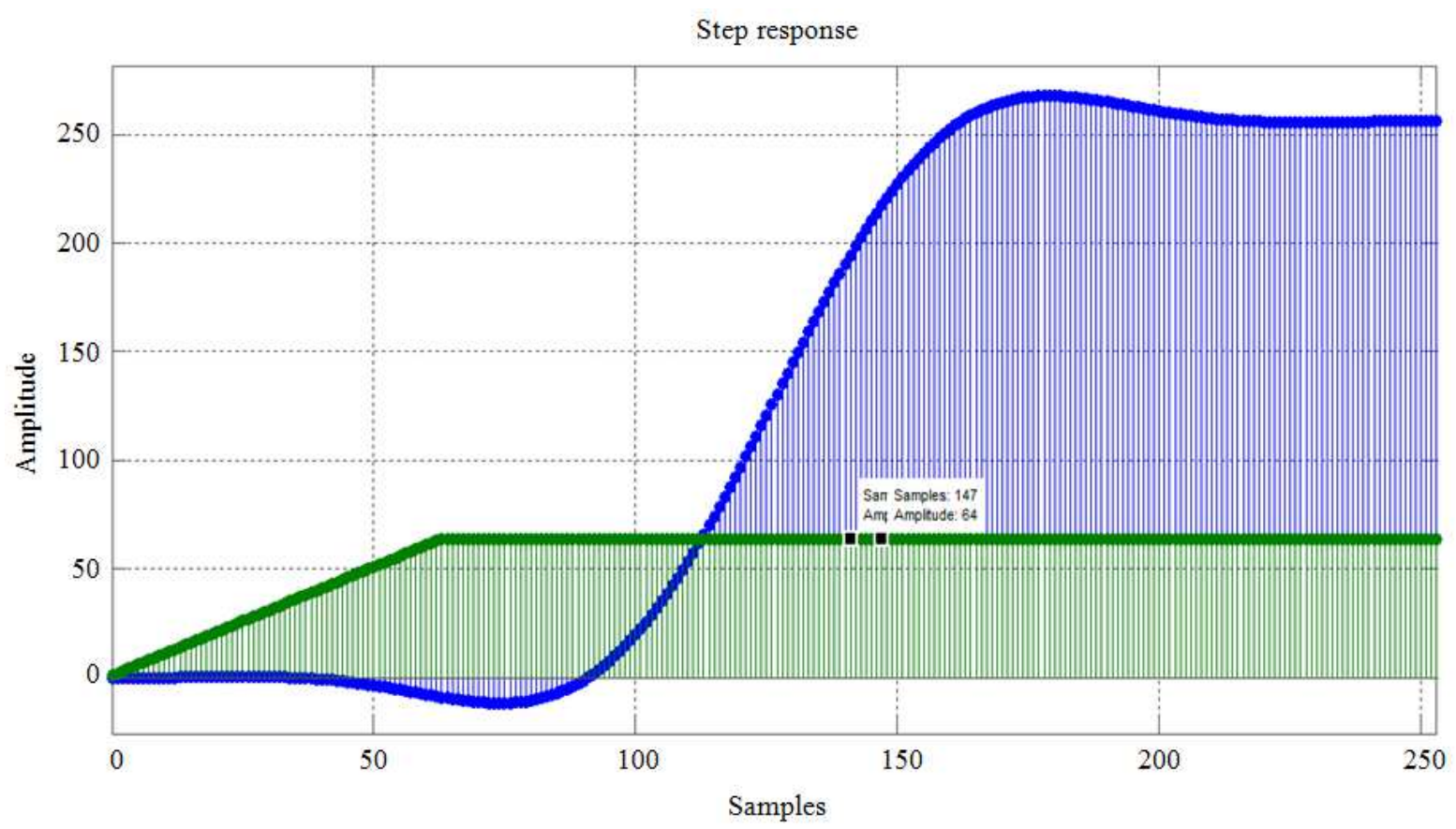

Fig. 3. Step Response of OFDM and FBMC: The Fig. 3. represent the step response of OFDM and FBMC

The Fig. 2, represent the magnitude response of the system for overlapping factor $(\mathrm{k}=2,3,4)$. As shown in above figure, the magnitude decrease with increasing overlapping factor.

As shown in Fig. 3, the amplitude of OFDM is vary with respect of frequency whereas the response of FBMC is flat with respect to frequency.

\section{Conclusion}

Although OFDM System have lot of advantages: reduction of ISI, increasing capacity and it easily integrate with multiple antenna hardware at both transmitter and receiver side but the insertion of cyclic prefix, its large side-lobes and peak-average-topower-ratio reduces the efficiency of OFDM System. For example in 4G mobile communication system: Out of $10 \mathrm{MHz}$ operating bandwidth, $7 \%$ of bandwidth is lost due to cyclic prefix. Hence FBMC is the most promising modulation scheme for $5 \mathrm{G}$ mobile communication system since it do not use $\mathrm{CP}$ and without wasting the bandwidth, the efficiency of FBMC is greatly enhanced as compared to OFDM. In this study prototype filter for FBMC and OFDM is designed and results show that FBMC outperforms OFDM.

\section{Funding Information}

Authors like to thanks JECRC University for funding and helping this work.

\section{Author's Contributions}

Arun Kumar: Designed and performed experiment, analysed data and wrote the paper.

Manisha Gupta: Have guided and designed the research plan.

\section{Ethics}

This article is original and contains unpublished material. The corresponding author confirms that all of the other authors have read and approved the manuscript and no ethical issues involved.

\section{References}

Berg, V., J.B. Doré and D. Noguet, 2014a. A flexible FS-FBMC receiver for dynamic access in the TVWS. Proceedings of the 9th International Conference on Cognitive Radio Oriented Wireless Networks and Communications, Jun. 2-4, IEEE Xplore Press, Oulu, pp: 285-290.

Berg, V., J.B. Doré and D. Noguet, 2014b. A multiuser FBMC receiver implementation for asynchronous frequency division multiple access. Proceedings of the 17th Euromicro Conference on Digital System Design, Aug. 27-29, IEEE Xplore Press, Verona, pp: 16-21. DOI: 10.1109/DSD.2014.11

Chang, R.W., 1996. Synthesis of band-limited orthogonal signals for multichannel data transmission. Bell Syst. Tech. J., 5: 1775-1797. 
Doelz, M.L., E.T. Hold and D.L. Martin, 1957. Binary data transmission techniques for linear systems. Proc. IRE, 45: 665-661.

Fang, J., Z. You, I.T. Lu, J. Li and R. Yang, 2013. Comparisons of filter bank multicarrier systems. Proceedings of the Long Island Systems, Applications and Technology Conference, May 3-3, IEEE Xplore Press, Farmingdale, NY, 1-6.

DOI: 10.1109/LISAT.2013.6578232

Han, S.H. and J.H. Lee, 2005. An overview of peak-toaverage power ratio reduction techniques for multicarrier transmission. IEEE Personal Commun., 12: 56-65. DOI: 10.1109/MWC.2005.1421929

He, Q. and A. Schmeink, 2015. Comparison and evaluation between FBMC and OFDM systems. Proceedings of the 19th International ITG Workshop on Smart Antennas WSA, Mar. 3-5, IEEE Xplore Press, Ilmenau, Germany, pp: 1-7.

Kumar, A. and M. Gupta, 2015. Design of OFDM and PAPR reduction using clipping method. Desidoc Drdo.

Lu, B. and X. Wang, 2000. Space time code design in OFDM system. Proceedings of the Globecom Global Telecommunications Conference, (GTC' 00), IEEE Xplore Press, San Francisco, CA, pp: 1000-1004. DOI: 10.1109/GLOCOM.2000.891288
Ndo, G., H. Lin and P. Siohan, 2012. FBMC/OQAM equalization: Exploiting the imaginary interference. Proceedings of the 23rd International Symposium on Personal Indoor and Mobile Radio Communications, Sept. 9-12, IEEE Xplore Press, Sydney, NSW, pp: 2359-2364. DOI: 10.1109/PIMRC.2012.6362751

Septh, M., S.A. Fechtel, G. Cock and H. Meyr, 1999. Optimum receiver design for wireless broad-band systems using OFDM. IEEE Trans. Commun., 47: 1668-1677. DOI: $10.1109 / 26.803501$

Zakariam, R. and D. Le Ruye, 2010. A novel FBMC scheme for spatial multiplexing with maximum likelihood detection. Proceedings of the 7th International Symposium on Wireless Communication Systems, Sept. 19-22, IEEE Xplore Press, York, pp: 461-465.

DOI: $10.1109 /$ ISWCS.2010.5624495 